\title{
Clinical Challenges in an Outbreak of COVID-19 in Wuhan, China: Concerns from Frontiers
}

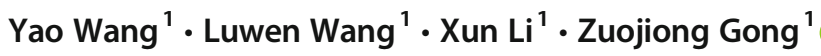 \\ Accepted: 9 September 2020 / Published online: 23 September 2020 \\ (C) Springer Science+Business Media, LLC, part of Springer Nature 2020
}

\begin{abstract}
Purpose of Review In December 2019, outbreaks of severe acute respiratory syndrome coronavirus 2 (SARS-CoV-2) infected pneumonia patients were discovered in Wuhan City, Hubei Province, China. With the spread of the epidemic, new cases have been found in other regions of China and abroad. This review summarizes the current coronavirus disease 2019 (COVID-19) transmission pathways, high-risk factors, diagnostic points, pathogenesis, and therapeutic drugs to provide the prevention and treatment theories for COVID-19.

Recent Findings COVID-19 is highly contagious and is transmitted mainly through the respiratory tract. Most patients with SARS-CoV-2 infection have milder symptoms. Some patients will have mild disease in the early stage, suddenly exacerbate later, and eventually die of multiple organ failure caused by cytokine storm. The basis for clinical diagnosis of COVID-19 is not just the detection of viral nucleic acids as the gold standard. The diagnostic accuracy improves when viral nucleic acid test is combined with clinical symptoms, CT results, and biochemical tests. For the treatment of COVID-19, the specific antiviral agents have not been developed, except for symptomatic supportive treatments.

Summary The methods for detecting SARS-CoV-2 infection have become increasingly mature, but specific antiviral drugs for the treatment of COVID-19 have not yet been developed. Treatment must pay attention to the cytokine storm that leads to the seriousness of COVID-19.
\end{abstract}

Keywords COVID-19 · SARS-CoV-2 · Clinical challenges

\section{Introduction}

Since December 2019, many cases of viral pneumonia have been found in Wuhan City, Hubei Province, China. It has been determined that the viral infection is transmitted from human to human, which seriously affects public health safety [1]. The World Health Organization (WHO) has named the new coronavirus that caused the Wuhan pneumonia epidemic for severe acute respiratory syndrome coronavirus 2 (SARS-CoV-

This article is part of the Topical Collection on Tropical, Travel and Emerging Infections

Zuojiong Gong

zjgong@163.com

1 Department of Infectious Diseases, Renmin Hospital of Wuhan University, Wuhan, China
2) and declared a pandemic. The new coronavirus-infected disease was named coronavirus disease 2019 (COVID-19).

SARS-CoV-2 belongs to the genus beta coronavirus and has higher homology with severe acute respiratory syndromes-associated coronavirus (SARS-CoV) than with Middle East respiratory syndrome-associated coronavirus (MERS-CoV) [2, 3]. SARS-CoV-2 is distinct from SARS$\mathrm{CoV}$, but the common outgroup with SARS-CoV is a HPU9-1 coronavirus parasitic to fruit bats [2]. It was recently found that the similarity between SARS-CoV-2 and RaTG13 isolated from Yunnan chrysanthemum bat was 96.2\% [4]. Therefore, the natural host of the virus is almost certainly bat. However, during the transmission of SARS-CoV-2 to humans by bats, snakes [5], mink [6], and pangolins may be intermediate hosts. The information about the intermediate host still awaits clarification. It is worth noting that asymptomatic infection may also be a potential way of transmission. Wuhan South China Seafood Market is currently considered 
to be the origin of SARS-CoV-2 [7]. However, it remains unclear if the South China Seafood Market is the sole source of the virus [8]. This review summarizes the current COVID19 transmission pathways, high-risk factors, diagnostic points, pathogenesis, and therapeutic drugs to provide a plan for the scientific control of COVID-19.

\section{Ways of Transmission}

The SARS-CoV-2 transmission routes are mainly direct transmission, aerosol transmission, and contact transmission. The public health information is still that droplets are the main route of transmission. The size of the droplet cores containing SARS-CoV-2 is in the sub-micron to micron range. Submicron range droplets necessarily imply aerosol transmission. Larger droplets, as one sees with droplet/contact transmission, are more in the $>10$ um range (lots of debate around the exact size of "droplets" that can aerosolize, but most would agree that those $<2$ um would certainly fall into that category of aerosolization). SARS-CoV-2 in the droplet core is protected by a protein envelope, which may remain active for a long time. If the droplet is aerosolized and inhaled by humans, it may cause infection. It is also worth noting that SARS-CoV-2 can be detected in the conjunctival sac of COVID-19 patients [9]. Meanwhile, an analysis of 9 pregnant women infected with COVID-19 found that there was no vertical transmission between mothers and infants [10].

\section{Characteristics of High-Risk Warning and Deaths}

A study involving 1099 patients in China found that all populations were susceptible to SARS-CoV-2, with an average age of 47.0 years $(35.0-58.0)$. The proportion of patients $\geq$ 65 yrs. was 153/1011 (15.1\%) [1]. Medical staff is also a highrisk group. The peak period for medical staff cases occurred on January 28. Among the 422 medical institutions providing diagnosis and treatment services for patients with COVID-19, a total of 3019 medical staff were infected with the new coronavirus (1716 confirmed cases), of whom 5 died and the crude case fatality rate was $0.3 \%$. There may be infections from nonoccupational exposures. These data confirmed that medical staffs were concentrated in Wuhan $(64 \%)$ and the rest of Hubei $(23.3 \%)$. The proportion of severe cases diagnosed by medical staff was $17.7 \%$ in Wuhan, $10.4 \%$ in Hubei, and $7.0 \%$ outside Hubei nationwide. On the whole, most cases were mild $(85.4 \%)$ [11].

The risk of COVID-19 death is mainly related to age, underlying chronic disease, and the time interval from the onset of initial symptoms to dyspnea [12]. The deaths caused by SARS-CoV-2 mainly occur in elderly patients and usually the elderly with underlying diseases [13]. A study of statistical analysis of public case data found that the median time from symptom onset to death was 13.8 days and the median time from hospitalization to death was 8.3 days [14]. The highest crude case fatality rate in the age group $\geq 80$ years was $14.8 \%$. For the risk of chronic disease, the cardiovascular mortality rate was $10.5 \%$, diabetes was $7.3 \%$, chronic respiratory disease was $6.3 \%$, hypertension was $6.0 \%$, and cancer was $5.6 \%$. Severe cases accounted for $13.8 \%$, and critical cases (includes both mechanically ventilated patients and anyone admitted to ICU) accounted for $4.7 \%$. The crude case fatality rate in critical cases was $49 \%$ [11].

\section{The Gold Standard for Diagnosing COVID-19: Virological Testing or Combined with Computed Tomography (CT)?}

WHO has launched the "Clinical management of severe acute respiratory infection when SARS-CoV-2 infection is suspected: Interim Guidance" (referred as "WHO guidelines") to strengthen outbreak control and patient management [15]. The gold standard for WHO guidelines emphasizes the SARS-CoV-2 nucleic acid testing. However, there were large numbers of suspected patients with clinical symptoms, pulmonary imaging findings, and abnormal routine blood tests, for whom the virological nucleic acid test is negative. Therefore, the National Health and Medical Commission of China issued the "Pneumonitis Diagnosis and Treatment Program for New Coronavirus Infection (Trial Version 5)" (referred as "Chinese Guideline") has re-defined the confirmed cases [16].

The patients in other provinces outside Hubei were still divided into "suspected cases" and "confirmed cases." However, the patients who have no clear epidemiological history but fulfilled 3 of the clinical manifestations, namely, "fever and/or respiratory symptoms," "imaging characteristics of pneumonia," and "the total number of white blood cells was normal or decreased, or the lymphocyte count was reduced in the early onset", could be defined as confirmed cases awaiting verification. The diagnostic criteria for confirmed cases require positive nucleic acid results.

It is particularly noteworthy that for cases in Hubei Province, a new category of "clinically diagnosed cases" has been added. Suspected cases with pneumonia imaging features are defined "clinically diagnosed cases." The "suspected case" standard is defined as a case that meets the two clinical manifestations of "fever and/or respiratory symptoms" and "normal or reduced white blood cell count in early onset, or decreased lymphocyte count" whether there is epidemiological link. In other words, the diagnostic criteria for suspected cases in Hubei Province have been relaxed. The diagnostic criteria for confirmed cases have not changed. 
As a result, the number of confirmed cases in Hubei Province dramatically rose to 14,840 on the second day after the Chinese Guideline being issued (that is, February 13, 2020). In our opinion, nucleic acid testing is no longer the gold standard for the diagnosis of COVID-19 infection. For the huge number patients with negative nucleic acid testing results, reasonable use of $\mathrm{CT}$ and clinical symptoms as a diagnostic basis is more conducive to the prevention and control of COVID-19.

\section{Possible Pathogenesis for COVID-19: Cytokine Storm}

The "cytokine storm" was first proposed in 1993 in graft versus host disease (GVHD) [17]. Pro-inflammatory factors include interleukin (IL), chemokines, colony-stimulating factors (CSFs), and tumor necrosis factor (TNF). When the immune system is activated due to infection, drugs, or autoimmune diseases, pro-inflammatory factors are activated and recruit other immune cells to secrete more cytokines, thus forming a positive feedback cycle. A large number of proinflammatory factors may be secreted, leading to unlimited positive feedback cycles, eventually resulting in cytokine storm [18]. Once cytokine storm occurs, it can quickly cause single or multiple organ failure and eventually threaten life [18].

The prediction of cytokine storm mainly depends on the detection of elevated inflammatory factors in blood. A retrospective study of 41 SARS-CoV-2 infected patients found that the expressions of IL-2, IL7, IL10, GSCF, IP10, MCP1, MIP1A, TNF $\alpha$, and other pro-inflammatory factors in plasma were significantly higher in ICU patients than in non-ICU patients [19]. An analysis of the status of cellular immunity and cytokines in 123 COVID-19 patients found that CD4 + T (52.90\% vs. $95.24 \%)$ and $\mathrm{CD} 8+\mathrm{T}(28.40 \%$ vs. $61.90 \%)$ were significantly lower in ICU patients than non-ICU patient. T lymphocytes are more likely to be suppressed in the severe patients [20]. The reduction of NK cells in non-ICU patient group was $34.31 \%$ and the ICU patient group was $47.62 \%$, indicating that the activity of NK cells was limited to some extent [20]. The IL4, IL10, IL17, and TNF of 21 patients in ICU patient group were all in the normal range. There was no significant difference between ICU and non-ICU patient groups for TNF and IFN levels. The proportion of IL6 in ICU patient group was significantly higher than that in nonICU patient group (76.19\% vs. $30.39 \%$ ) [20]. These data on inflammatory indicators suggest a cytokine storm in ICU patients, resulting in a worse overall prognosis.

For the specific process of cytokine storm (Fig. 1), SARSCoV-2 infects lung epithelial cells by binding with ACE2 receptors [21]. Cell death, apoptosis, and pyroptosis occur due to infection-induced production of pro-inflammatory cytokines (IL1 $\beta$, IL6, IL8) and chemokines (IP10, MCP1) $[19,22]$. These factors mediate the early recruitment of monocytes/macrophages in lung and promote the production of a unique set of chemokines by activated macrophages, such as MIG, IP-10, and MCP-1 [19]. These chemokines further recruit neutrophils, monocytes, and $\mathrm{T}$ cells into lung tissue [23]. Activated $\mathrm{T}$ cells then migrate into the lung tissue and specifically destroy virus-infected lung cells by releasing perforin $[19,23]$. It could lead to extensive tissue damage to the lung parenchyma and ARDS [24]. On the other hand, SARS-CoV-2 rapidly activates pathogenic $\mathrm{T}$ cells to produce cytokines such as granulocyte-macrophage colony-stimulating factors (GM-CSF) and IL6. GM-CSF will further activate $\mathrm{CD} 14+\mathrm{CD} 16+$ inflammatory monocytes and produce a larger amount of IL6 and other inflammatory factors [25]. Thereby, it forms a cytokine storm, leading to severe immune damage to the lungs and other organs. In summary, the levels of CD $4+\mathrm{T}$ and CD8 $+\mathrm{T}$ are generally low; the level of IL- 6 is high in the severe patients. However, the "warning value" of CD4+ T, CD8 + T, and IL-6 still needs a large number of samples to confirm.

The main methods to treat cytokine storm are to eliminate the pathogens and suppress inflammatory responses. Specific drugs against SARS-CoV-2 are still in clinical trials, which will take several months to produce results [26]. Although the application of glucocorticoids can inhibit normal human immunity and delay the removal of coronavirus, for the severe patients caused by cytokine storm, the most commonly used and effective drug to directly reduce cytokine storm is still glucocorticoids [27, 28]. In addition, immunosuppressive agents, such as anti-IL-6 monoclonal antibody, gamma globulin, PPARs inhibitors, and sphingosine phosphate 1 (S1P1) receptors inhibitors, cyclooxygenase (COX) inhibitors, antioxidants, and anti-tumor necrosis factor therapy may be effective against cytokine storm [29, 30]. Although most cases have mild symptoms and good prognosis, cytokine storm is still a hidden danger in the mild patients with COVID-19. Early monitoring and early treatment are the best ways to eliminate this hidden danger.

\section{Development and Evaluation of Antiviral Drugs}

The development of vaccines, monoclonal antibodies, oligonucleotide-based therapies, peptides, interferon treatments, and small-molecule drugs for COVID-19 will take at least months or even years. Therefore, it is recommended to find antiviral drugs with therapeutic potential among the drugs approved in the market for the treatment of human immunodeficiency virus (HIV), hepatitis B virus (HBV), hepatitis C virus (HCV), severe acute respiratory syndrome (SARS), and Middle East respiratory syndrome (MERS). Nucleoside 


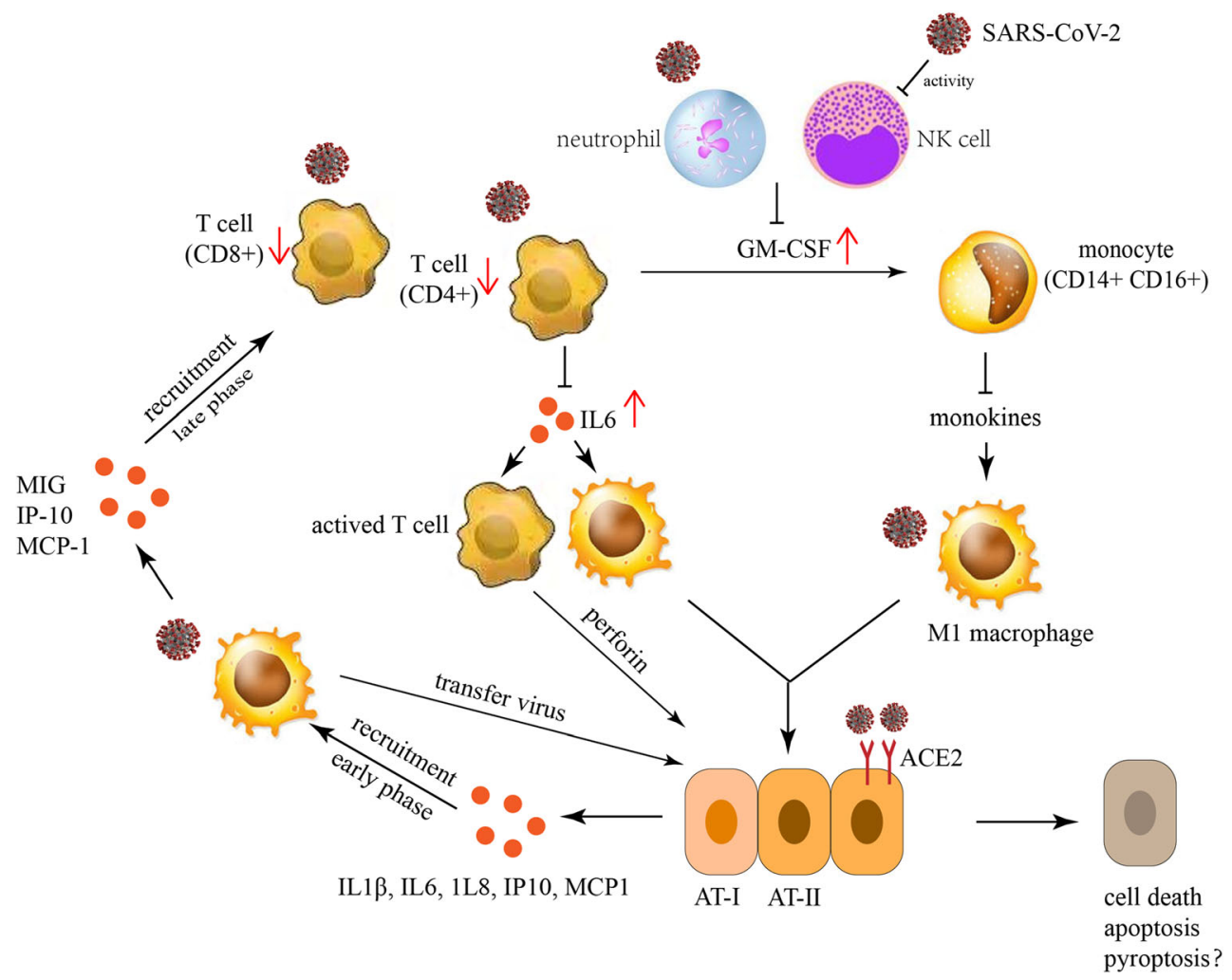

Fig. 1 A model of SARS-CoV-2 pathogenesis. For the specific process of cytokine storm (Fig. 1), SARS-CoV-2 infects lung cells epithelial cells by binding with ACE2 receptors. Death, apoptosis, and pyroptosis of infected cells due to infection induces the production of proinflammatory cytokines (IL1 $\beta$, IL6, IL8) and chemokines (IP10, MCP1). These factors mediate the early recruitment of monocytes/ macrophages in lung and promote the production of a unique set of chemokines by activated macrophages, such as MIG, IP-10, and MCP1. These chemokines further recruit neutrophils, monocytes, and T cells into lung tissue. Activated T cells then migrate into the lung tissue and

analogs such as favipiravir, ribavirin, remdesivir, and galidesivir can block RNA synthesis of various RNA viruses including SARS-CoV-2 through targeted intervention with RNA polymerase [31]. Approved protease inhibitors, including disulfiram, lopinavir, and ritonavir, have activity against MERS-CoV and SARS-CoV [26].

\section{Favipiravir}

Favipiravir is a guanine analog approved for the treatment of influenza that effectively inhibits RNA viruses such as influenza virus, Ebola virus, yellow fever virus, chikungunya virus, norovirus, and enterovirus [31]. Favipiravir is a nucleoside antiviral drug, and its mechanism of action has not been fully elucidated. Its target is a viral RNA-dependent RNA polymerase. After oral absorption, the drug is converted into a nucleoside triphosphate of biologically active favipiravir. Its structure is similar to purine, and it can compete with purine for virus RNA polymerase. Favipiravir's nucleoside triphosphates can also be inserted into the viral RNA chain and specifically destroy virus-infected lung cells by releasing perforin. It could lead to extensive tissue damage to the lung parenchyma and ARDS. On the other hand, SARS-CoV-2 rapidly activates pathogenic $\mathrm{T}$ cells to produce cytokines such as granulocyte-macrophage colonystimulating factors (GM-CSF) and IL6. GM-CSF will further activate CD14 + CD16+ inflammatory monocytes and produce a larger amount of IL6 and other inflammatory factors. Thereby, it forms a cytokine storm, leading to severe immune damage to the lungs and other organs. AT-I, alveolar type I cell. AT-II, alveolar type II cell

induce fatal mutations in the virus [32]. Therefore, mechanistically, favipiravir has potential antiviral effects on various RNA viruses. The steady-state trough concentration of favipiravir is $11.3-22.6 \mu \mathrm{g} / \mathrm{mL}$. It has been reported that favipiravir has anti-SARS-CoV-2 activity in Vero E6 cells, with an EC50 of $61.88 \mu \mathrm{M}$ [33].

\section{Remdesivir}

Remdesivir is a modified prodrug of the adenine nucleoside analog. It has strong in vitro activity and cell permeability and can be more effectively metabolized into active nucleoside triphosphate (NTP). NTP acts as a substrate for RNA polymerase in viral RNA polymerase-guided primer extension, thereby terminating the transcription of RNA strand information [34]. Coronavirus expresses exonuclease in nonstructural protein 14, nsp14, and it is conserved across the entire coronavirus family. Nucleoside analogs are incorporated into viral RNA and are excised by coronavirus exonucleasees [35]. However, remdesivir is resistant to 
exonucleases, which is more effective than other nucleoside analogs [36]. Remdesivir has broad-spectrum antiviral activity against RNA viruses. Remdesivir can effectively inhibit the replication of SARS-CoV and MERS-CoV coronavirus, and the inhibitory effect on coronavirus has a dose-dependent effect. It has preventive therapeutic effect on coronavirus and can reduce the symptoms shown in experimental animals after SARS-CoV infection, reduce virus titer in the lungs, and thus reduce lung tissue damage [37]. SARS-CoV-2 is similar to SARS-CoV and MERS-CoV in terms of structure and genome, as well as clinical manifestations of infection. In in vitro and animal models, data confirm its role as an antiviral against coronavirus. However, clinical data on remdesivir for the treatment of patients with coronavirus infection are very limited. A recent study reported that remdesivir could inhibit SARS-CoV-2 (EC50 in Vero E6 cells $=0.77 \mu \mathrm{M})$ [33]. A patient with SARS-CoV-2 in the USA recovered after receiving intravenous remdesivir [38]. It is currently in early clinical studies to evaluate the safety and effects of galidesivir in COVID-19 patients.

\section{Lopinavir/Ritonavir}

Lopinavir/ritonavir is a class of Mpro (3CLpro) inhibitors and is considered to be a 3-chymotrypsin-like protease that inhibits MERS-CoV and SARS-CoV. Compared with ribavirin alone, the combined use of lopinavir/ritonavir significantly improved the prognosis of SARS-CoV patients [39]. In vitro models of MERS-CoV infection demonstrate that lopinavir/ ritonavir has no significant antiviral activity and it is rarely used in the treatment of patients with MERS-CoV. However, another study reveals that lopinavir/ritonavir is effective when the MERS marmoset model was infected with MERS-CoV. The prognosis of marmoset could be significantly improved. A clinical trial of 199 COVID-19 patients found that the effectiveness of lopinavir/ritonavir treatment could not be observed compared to conventional treatment [40]. Moreover, the combination of interferon (IFN)- $\beta 1 \mathrm{~b}$ achieved better curative effect [41]. It is important to pay attention to lopinavir/ritonavir-related adverse reactions and interaction with other drugs.

\section{Chloroquine}

Chloroquine has several possible antiviral mechanisms. As a weakly basic drug, chloroquine enters cells in a nonprotonated form in extracellular fluid and becomes a protonated form in acidic environments such as endosomes, Golgi apparatus, and lysosomes. The alkaline phagosome environment interferes with numerous metabolic enzymes [42, 43]. Because many viruses need to enter cells through phagocytosis under acidic conditions at lower $\mathrm{pH}$, chloroquine can inhibit the penetration, husking, and replication of viruses. For enveloped viruses, processes such as glycoprotein translation modification also require a low $\mathrm{pH}$ environment, and chloroquine can inhibit a variety of viruses through its $\mathrm{pH}$ dependence [44]. For some viruses of the Flaviviridae family, chloroquine can also inhibit the hydrolysis of its proteins and disrupt the viral infection process; chloroquine can also inhibit some non-infectious retroviruses by inhibiting the glycosylation of viral envelope proteins [42]. In addition, the virus needs to attach to cell receptors through mucin to infect cells. ACE-2 was found to be a receptor protein for SARS-CoV and SARS-CoV-2. SARS-CoV-2 enters cells through the ACE-2 receptor. Chloroquine may interfere with the virus. The binding of mucin to cellular ACE-2 receptor protein exerts an inhibitory effect on the virus [45]. In addition to its direct antiviral effect, chloroquine can also regulate immune activity and inhibit inflammatory reactions associated with viral infections [46]. Therefore, it is often used in clinical practice to treat immune system diseases such as rheumatoid arthritis. Immune system diseases often have overexpression of inflammatory cytokines. Chloroquine inhibits the inflammatory response by reducing the expression of inflammatory cytokines such as TNF- $\alpha$. This may have a positive effect on the activation of the immune system and the severe inflammatory response accompanied by the viral infection [42]. Chloroquine may also be used to treat lung damage caused by viral infection by inhibiting cell autophagy [47].

\section{Hydroxychloroquine}

The difference between hydroxychloroquine and chloroquine is that an ethyl group in chloroquine is replaced by a hydroxyethyl group in hydroxychloroquine [48]. The therapeutic effect is similar, but the side effects are significantly reduced compared with chloroquine. Therefore, hydroxychloroquine is widely used as an immunosuppressive agent in the treatment of rheumatic diseases [48]. By observing the electrocardiogram of 84 COVID-19 patients who took oral hydroxychloroquine and azithromycin for 5 days, it was found that most of the patients who received hydroxychloroquine and azithromycin showed an extended QT interval. Moreover, the severity of other underlying diseases and SARS-CoV-2 infection may aggravate this situation [49].

\section{Respiratory Support Therapy}

Some patients with COVID-19 can progress rapidly to acute respiratory distress syndrome (ARDS) $[16,50]$. Among them, severe hypoxemia is a common clinical manifestation of such patients and usually requires respiratory support treatment in the intensive care unit [51]. Patients with severe COVID-19 need individualized respiratory support technology based on the degree of hypoxia in the patient. For patients with mild to 
moderate hypoxia, nasal catheters, masks, nasal high-flow oxygen therapy, and non-invasive ventilation can be preferred for non-invasive ventilation. Self-pronation can also ameliorate blood oxygenation and is a low-tech intervention that can prevent the need for more invasive methods of oxygen delivery. Awake pronation is also used in ICU and other inpatient populations, but it has saved many an emergency department (ED) patient from going on the vent. Non-invasive ventilation, invasive mechanical ventilation, and external membrane oxygenation (ECMO) can be selected for moderate to severe hypoxia. Severe COVID-19 requires mechanical ventilation in the event of severe hypoxemia and respiratory distress. Clinical studies on MERS-CoV, SARS-CoV, and influenza have shown that non-invasive ventilation has a higher failure rate in such patients [15]. Delayed intubation can increase patient mortality [52]. Compared with SARS-CoV, the progression of respiratory failure in COVID-19 patients is slower. Some patients with severe respiratory failure can still be successfully treated with non-invasive ventilation. It is recommended to use non-invasive ventilation as much as possible. When patients with impaired consciousness level, upper airway obstruction, deterioration of oxygenation, or deterioration of ventilation status [53], invasive artificial airway invasive ventilation should be established immediately.

\section{Prevention and Control of Nosocomial Infection}

An analysis of data from 138 SARS-CoV-2 cases found that 40 medical staff (29\%) and 17 hospitalized patients $(12.3 \%)$ had nosocomial infection in the hospital. In the infected medical staff, 31 worked in the general ward, 7 in the emergency department, and 2 in the intensive care unit [54]. The invading power of the virus from "super communicators" cannot be underestimated. In addition, patients with heart disease and diabetes have a low ability to defend themselves against infections. As mentioned above, the best way to prevent nosocomial infection in hospitals is to establish and implement a strict disinfection and isolation system, including airway management, quarantine measures, and disposal of excreta.

\section{Conclusions}

COVID-19 is highly contagious and is transmitted mainly through the respiratory tract. Most patients with SARS-CoV2 infection have milder symptoms. Some patients will have mild disease in the early stage, suddenly exacerbate later, and eventually die of multiple organ failure, which must get worldwide attention. It is worth noting that some asymptomatic patients are also potential sources of infection. The diagnostic criteria for cytokine storm caused by SARS-CoV-2 need to be further studied. The diagnosis of COVID-19 relies on the detection of viral nucleic acids as the gold standard. However, diagnostic accuracy is better when combined with clinical symptoms, CT results, and biochemical tests. At present, for the treatment of COVID-19, no specific antiviral agents have been developed, except for symptomatic supportive treatments. Therefore, based on the present information, the strategies for the prevention and treatment of COVID-19 need be further studied.

Authors' Contribution Zuojiong Gong made substantial contributions to the study concept and design. Yao Wang wrote the manuscript draft. Yao Wang, Luwen Wang, and Xun Li collected the references. Zuojiong Gong made substantial revisions to the manuscript.

Funding The current work was supported by the National Natural Science Foundation Project of China (Grant No. 81870413).

\section{Compliance with Ethical Standards}

Conflict of Interest The authors declare that they have no conflict of interests.

Ethical Approval Not required.

Human and Animal Rights and Informed Consent This article does not contain any studies with human or animal subjects performed by any of the authors.

\section{References}

1. Guan W-j, Ni Z-y, Hu Y, Liang W-h, Ou C-q, He J-x, Liu L, Shan H, Lei C-1, Hui DS, et al. Clinical characteristics of 2019 novel coronavirus infection in China. medRxiv. 2020. 2020.2002.2006.20020974.

2. Xu X, Chen P,Wang J, Feng J, Zhou H, Li X, et al. Evolution of the novel coronavirus from the ongoing Wuhan outbreak and modeling of its spike protein for risk of human transmission. Sci China Life Sci. 2020;63:457-60.

3. Zhu N, Zhang D, Wang W, Li X, Yang B, Song J, et al. A novel coronavirus from patients with pneumonia in China, 2019. N Engl J Med. 2020;382:727-33.

4. Zhou P, Yang XL, Wang XG, Hu B, Zhang L, Zhang W, et al. A pneumonia outbreak associated with a new coronavirus of probable bat origin. Nature. 2020;579:270-73.

5. Ji W, Wang W, Zhao X, Zai J, Li X. Cross-species transmission of the newly identified coronavirus 2019-nCoV.J Med Virol. 2020;92: 433-40.

6. Guo Q, Li M, Wang C, Wang P, Fang Z, Tan J, Wu S, Xiao Y, Zhu H. Host and infectivity prediction of Wuhan 2019 novel coronavirus using deep learning algorithm. bioRxiv. 2020 . 2020.2001.2021.914044.

7. Chan JF, Yuan S, Kok KH, To KK, Chu H, Yang J, et al. A familial cluster of pneumonia associated with the 2019 novel coronavirus indicating person-to-person transmission: a study of a family cluster. Lancet. 2020;395:514-23.

8. Jon Cohen. Wuhan seafood market may not be source of novel virus spreading globally. 2020. https://doi.org/10.1126/science. abb0611. 
9. Zhou Y, Zeng Y, Tong Y, Chen C. Ophthalmologic evidence against the interpersonal transmission of 2019 novel coronavirus through conjunctiva. medRxiv. 2020. 2020.2002.2011.20021956.

10. Chen H, Guo J, Wang C, Luo F, Yu X, Zhang W, et al. Clinical characteristics and intrauterine vertical transmission potential of COVID-19 infection in nine pregnant women: a retrospective review of medical records. Lancet. 2020;395:809-15. https://doi.org/ 10.1016/S0140-6736(20)30360-3.

11. The Novel Coronavirus Pneumonia Emergency Response Epidemiology Team. Analysis of epidemiological characteristics of new coronavirus pneumonia. Chin J Epidemiol. 2020;41:14551.

12. Kui L, Fang YY, Deng Y, LiuW, WangMF,Ma JP, et al. Clinical characteristics of novel coronavirus cases in tertiary hospitals in Hubei Province. Chin Med J (Engl). 2020;133:1025-31.

13. Shaman J, Galanti M. Direct measurement of rates of asymptomatic infection and clinical care-seeking for seasonal coronavirus. medRxiv. 2020. 2020.2001.2030.20019612.

14. Linton NM, Kobayashi T, Yang Y, Hayashi K, Akhmetzhanov AR, Jung S-m, Yuan B, Kinoshita R, Nishiura H. Epidemiological characteristics of novel coronavirus infection: a statistical analysis of publicly available case data. medRxiv. 2020 . 2020.2001.2026.20018754.

15. World Health Organization: clinical management of severe acute respiratory infection when novel coronavirus $(\mathrm{nCoV})$ infection is suspected: Interim Guidance.

16. Diagnosis and treatment of pneumonia caused by a new coronavirus (trial version 5). Chin J Integr Med. 2020:1-3 http://kns.cnki. net $/ \mathrm{kcms} /$ detail/11.2787.R.20200208.1034.002.html. Accessed 21 Aug 2020.

17. Ferrara JL, Abhyankar S, Gilliland DG. Cytokine storm of graftversus-host disease: a critical effector role for interleukin-1. Transplant Proc. 1993;25:1216-7.

18. Tisoncik JR, Korth MJ, Simmons CP, Farrar J, Martin TR, Katze MG. Into the eye of the cytokine storm. Microbiol Mol Biol Rev. 2012;76:16-32.

19. Huang $\mathrm{C}$, Wang $\mathrm{Y}$, Li X, Ren L, Zhao J, Hu Y, et al. Clinical features of patients infected with 2019 novel coronavirus in Wuhan, China. Lancet. 2020;395:497-506.

20. Wan S, Yi Q, Fan S, Lv J, Zhang X, Guo L, Lang C, Xiao Q, Xiao $\mathrm{K}$, Yi Z, et al. Characteristics of lymphocyte subsets and cytokines in peripheral blood of 123 hospitalized patients with 2019 novel coronavirus pneumonia (NCP). medRxiv. 2020. 2020.2002.2010.20021832.

21. Cheng H, Wang Y, Wang GQ. Organ-protective effect of angiotensin-converting enzyme 2 and its effect on the prognosis of COVID-19. J Med Virol. 2020;92:726-30.

22. Yang Y, Peng F, Wang R, Guan K, Jiang T, Xu G, et al. The deadly coronaviruses: the 2003 SARS pandemic and the 2020 novel coronavirus epidemic in China. J Autoimmun. 2020;109:102434.

23. Liu J, Li S, Liu J, Liang B, Wang X, Wang H, et al. Longitudinal characteristics of lymphocyte responses and cytokine profiles in the peripheral blood of SARS-CoV-2 infected patients. EBioMedicine. 2020;55:102763.

24. Chen J, Subbarao K. The Immunobiology of SARS*. Annu Rev Immunol. 2007;25:443-72.

25. Zhou Y, Fu B, Zheng X, Wang D, Zhao C, Qi Y, Sun R, Tian Z, Xu $\mathrm{X}$, Wei H. Aberrant pathogenic GM-CSF+ T cells and inflammatory CD14+CD16+ monocytes in severe pulmonary syndrome patients of a new coronavirus. bioRxiv. 2020 . 2020.2002.2012.945576.

26. Li G, Clercq ED. Therapeutic options for the 2019 novel coronavirus (2019-nCoV). Nat Rev Drug Discov. 2020;19:149-50.

27. Chen RC, Tang XP, Tan SY, Liang BL, Wan ZY, Fang JQ, et al. Treatment of severe acute respiratory syndrome with glucosteroids: the Guangzhou experience. Chest. 2006;129:1441-52.
28. Zhao JP, Hu Y, Du RH, Chen ZS, Jin Y, Zhou M, et al. Expert consensus on the use of corticosteroid in patients with 2019-nCoV pneumonia. Zhonghua Jie He He Hu Xi Za Zhi. 2020;43:E007.

29. Fang Y, Zhang H, Xu Y, Xie J, Pang P, JiW. CT manifestations of two cases of 2019 novel coronavirus (2019-nCoV) pneumonia. Radiology. 2020;295:208-9.

30. Liu Q, Zhou YH, Yang ZQ. The cytokine storm of severe influenza and development of immunomodulatory therapy. Cell Mol Immunol. 2016;13:3-10.

31. De Clercq E. New nucleoside analogues for the treatment of hemorrhagic fever virus infections. Chem Asian J. 2019;14:3962-8.

32. Furuta Y, Komeno T, Nakamura T. Favipiravir (T-705), a broad spectrum inhibitor of viral RNA polymerase. Proc Jpn Acad Ser B Phys Biol Sci. 2017;93:449-63.

33. Wang M, Cao R, Zhang L, Yang X, Liu J, Xu M, et al. Remdesivir and chloroquine effectively inhibit the recently emerged novel coronavirus (2019-nCoV) in vitro. Cell Res. 2020;30:269-71.

34. Cho A, Saunders OL, Butler T, Zhang L, Xu J, Vela JE, et al. Synthesis and antiviral activity of a series of 1'-substituted 4-aza7,9-dideazaadenosine C-nucleosides. Bioorg Med Chem Lett. 2012;22:2705-7.

35. Jain J, Gaur S, Chaudhary Y, Kaul R. The molecular biology of intracellular events during coronavirus infection cycle. Virusdisease. 2020;31:1-5.

36. de Wilde AH, Snijder EJ, Kikkert M, van Hemert MJ. Host factors in coronavirus replication. Curr Top Microbiol Immunol. 2018;419:1-42.

37. Sheahan TP, Sims AC, Graham RL, Menachery VD, Gralinski LE, Case JB, et al. Broad-spectrum antiviral GS-5734 inhibits both epidemic and zoonotic coronaviruses. Sci Transl Med. 2017;9: eaal3653.

38. Holshue ML, DeBolt C, Lindquist S, Lofy KH, Wiesman J, Bruce $\mathrm{H}$, et al. First case of 2019 novel coronavirus in the United States. N Engl J Med. 2020;382:929-36.

39. Chu CM, Cheng VC, Hung IF, Wong MM, Chan KH, Chan KS, et al. Role of lopinavir/ritonavir in the treatment of SARS: initial virological and clinical findings. Thorax. 2004;59:252-6.

40. Cao B, Wang Y, Wen D, Liu W, Wang J, Fan G, et al. A trial of Lopinavir-ritonavir in adults hospitalized with severe Covid-19. N Engl J Med. 2020;382:1787-99.

41. Chan JF, Yao Y, Yeung ML, Deng W, Bao L, Jia L, et al. Treatment with lopinavir/ritonavir or interferon-betalb improves outcome of MERS-CoV infection in a nonhuman primate model of common marmoset. J Infect Dis. 2015;212:1904-13.

42. Savarino A, Boelaert JR, Cassone A, Majori G, Cauda R. Effects of chloroquine on viral infections: an old drug against today's diseases? Lancet Infect Dis. 2003;3:722-7.

43. Vincent MJ, Bergeron E, Benjannet S, Erickson BR, Rollin PE, Ksiazek TG, et al. Chloroquine is a potent inhibitor of SARS coronavirus infection and spread. Virol J. 2005;2:69.

44. Colson P, Rolain JM, Raoult D. Chloroquine for the 2019 novel coronavirus SARS-CoV-2. Int J Antimicrob Agents. 2020;55: 105923.

45. Li JY, You Z, Wang Q, Zhou ZJ, Qiu Y, Luo R, et al. The epidemic of 2019-novel-coronavirus (2019-nCoV) pneumonia and insights for emerging infectious diseases in the future. Microbes Infect. 2020;22:80-5.

46. Keyaerts E, Vijgen L, Maes P, Neyts J, Van Ranst M. In vitro inhibition of severe acute respiratory syndrome coronavirus by chloroquine. Biochem Biophys Res Commun. 2004;323:264-8.

47. Yan Y, Zou Z, Sun Y, Li X, Xu KF, Wei Y, et al. Anti-malaria drug chloroquine is highly effective in treating avian influenza A H5N1 virus infection in an animal model. Cell Res. 2013;23:300-2.

48. Shippey EA, Wagler VD, Collamer AN. Hydroxychloroquine: an old drug with new relevance. Cleve Clin J Med. 2018;85:459-67. 
49. Chorin E, Wadhwani L, Magnani S, Dai M, Shulman E, Nadeau Routhier C, et al. QT interval prolongation and torsade De Pointes in patients with COVID-19 treated with hydroxychloroquine/ azithromycin. Heart Rhythm. 2020;17:1425-33.

50. Chen N, Zhou M, Dong X, Qu J, Gong F, Han Y, et al. Epidemiological and clinical characteristics of 99 cases of 2019 novel coronavirus pneumonia in Wuhan, China: a descriptive study. Lancet. 2020;395:507-13.

51. Flower L, Martin D. Management of hypoxaemia in the critically ill patient. Br J Hosp Med. 2020;81:1-10.

52. Antonelli M, Conti G, Moro ML, Esquinas A, Gonzalez-Diaz G, Confalonieri $\mathrm{M}$, et al. Predictors of failure of noninvasive positive pressure ventilation in patients with acute hypoxemic respiratory failure: a multi-center study. Intensive Care Med. 2001;27:171828.

53. Cooper A, Joglekar A, Adhikari N. A practical approach to airway management in patients with SARS. CMAJ. 2003;169:785-7.

54. Wang D, Hu B, Hu C, Zhu F, Liu X, Zhang J, et al. Clinical characteristics of 138 hospitalized patients with 2019 novel coronavirus-infected pneumonia in Wuhan, China. JAMA. 2020;323: 1061-69.

Publisher's Note Springer Nature remains neutral with regard to jurisdictional claims in published maps and institutional affiliations. 